\title{
A INFLUÊNCIA DA VIA DE ACESSO NA LUXAÇÃO DAS ARTROPLASTIAS TOTAIS DO QUADRIL
}

\author{
THE INFLUENCE OF THE SURGICAL APPROACH CONCERNING \\ DISLOCATION IN TOTAL HIP ARTHROPLASTY
}

José Ricardo Negreiros Vicente', André Fernandes Pires², Bruno Takasaki Lee ${ }^{2}$, Marcos Camargo Leonhardł ${ }^{3}$, Leandro Ejnisman ${ }^{4}$, Alberto Tesconi Croci ${ }^{5}$

\section{RESUMO}

Objetivo: Avaliar a ocorrência de luxação nas artroplastias totais do quadril não cimentadas, comparando-se os acessos posterior e lateral direto. Métodos: Estudo retrospectivo comparativo com 232 pacientes submetidos à artroplastia total do quadril não cimentada, por diagnóstico de osteoartrose primária e secundária, sendo 105 submetidos ao acesso posterior e 127 ao acesso lateral. O modelo protético utilizado foi único, assim como o protocolo de reabilitação e cuidados pós-operatórios. Foram verificados ocorrência de luxação, posicionamento acetabular e componentes utilizados. Resultados: Ocorreu um caso isolado de luxação no grupo do acesso lateral direto $(0,8 \%)$ contra $0 \%$ no grupo de pacientes submetidos ao acesso posterior, sem diferença significativa $(p=1)$. A luxação ocorreu em uma paciente de 47 anos de idade, que foi tratada com redução incruenta sem recidiva da luxação após três anos e um mês de seguimento. O tempo de seguimento dos dois grupos variou entre seis e 42 meses, com média de 23,7 meses. Conclusão: Os autores concluem que a prevalência de luxação nas artroplastias totais do quadril é similar nos dois acessos estudados e que medidas educativas, técnica cirúrgica adequada e utilização de próteses com offsets maiores podem diminuir o risco dessa complicação.

Descritores - Quadril; Artroplastia; Luxação do quadril

\section{ABSTRACT}

Objectives: Our primary aim was to evaluate the occurrence of dislocation of non-cemented total hip arthroplasty, when using the posterior and the direct lateral approaches. Methods: We performed a comparative retrospective study with 232 patients submitted to non-cemented total hip arthroplasty, due to the diagnosis of primary or secondary osteoarthritis. The posterior approach was used in 105 patients while direct lateral approach was used in 127 patients. There was only one prosthesis model and the same rehabilitation program and post-operative care was used for all patients. We checked the occurrence of dislocation, the acetabular positioning and also the size of the components. Results: There was only one case of dislocation, treated with closed reduction successfully. This was a 47 year-old female, submitted to direct lateral approach. The mean follow-up time for both groups was 23.7 months, ranging from six to 42 months. Conclusion: The authors conclude that the prevalence of total hip arthroplasty dislocation is similar for both approaches, and educational measures besides the use of a higher femoral offset seem to reduce the risk of this complication.

Keywords - Hip; Arthroplasty; Hip dislocation

\footnotetext{
1 - Professor Colaborador da Faculdade de Medicina da USP e Assistente do Grupo de Quadril e Artroplastias do Instituto de Ortopedia e Traumatologia do HC/FMUSP.

2 - Residente do Instituto de Ortopedia e Traumatologia do HC/FMUSP.

3 - Assistente Voluntário do Grupo de Quadril e Artroplastias do Instituto de Ortopedia e Traumatologia do HC/FMUSP.

4 - Médico de Complementação Especializada do Grupo de Quadril e Artroplastias do Instituto de Ortopedia e Traumatologia do HC/FMUSP.

5 - Professor Associado da Faculdade de Medicina da USP e Chefe do Grupo de Quadril e Artroplastias do Instituto de Ortopedia e Traumatologia do HC/FMUSP.
}

Trabalho realizado no Instituto de Ortopedia e Traumatologia do HC/FMUSP.

Correspondência: Rua Afonso de Freitas, 488, ap. 22 -04006-052 - São Paulo, SP. E-mail: rrnegreiros@gmail.com 


\section{INTRODUÇÃO}

A artroplastia total do quadril é uma técnica cirúrgica que visa o alivio da dor articular e restauração funcional, sendo realizada amplamente desde a década de 60 após divulgação por Sir John Charnley ${ }^{(1)}$.

Entre as complicações advindas da técnica, a luxação pode ser considerada uma complicação peculiar a esse tipo de cirurgia; as outras complicações são frequentes em todos os tipos de cirurgia. Além do sofrimento que acarreta ao paciente com a possibilidade de nova intervenção cirúrgica, devemos considerar também o custo socioeconômico, podendo elevar o valor habitual em $50 \%{ }^{(2)}$.

A prevalência da luxação nas artroplastias totais do quadril é variável, com relatos de $0,43 \%$ a $6,9 \% \%^{(3,4)}$. O acesso cirúrgico é um dos principais fatores atribuídos à prevalência dessa complicação. Os trabalhos mais antigos consideravam o acesso posterior mais instável que o acesso lateral ${ }^{(5)}$. Porém, estudos recentes de maior evidência clínica através de revisão sistemática são inconclusivos quanto à influência do acesso cirúrgico sobre as luxações ${ }^{(6)}$.

O objetivo central do nosso estudo foi avaliar a ocorrência de luxação nas artroplastia totais do quadril, comparando-se os acessos posterior e lateral direto, utilizando-se um modelo único de prótese não cimentada.

\section{MÉTODOS}

Realizamos um estudo retrospectivo comparativo com 232 pacientes submetidos à artroplastia total do quadril não cimentada no Instituto de Ortopedia e Traumatologia do Hospital das Clinicas da Faculdade de Medicina da USP entre janeiro de 2006 e dezembro de 2008, comparando-se os acessos lateral direto e posterior. Trata-se de um estudo bicaudal, sendo nossa hipótese nula $\mathrm{H} 0$ a igualdade da ocorrência de luxação protética entre os dois grupos de pacientes e nossa hipótese alternativa $\mathrm{H} 1$ a diferença dessa ocorrência.

Foram incluídos no estudo todos os pacientes operados nesse período por diagnóstico de osteoartrose primária ou secundária, onde se utilizou um modelo único de prótese para todos os casos. O grupo de pacientes submetidos ao acesso posterior totalizou 105 operados por um único cirurgião (JRNV) e o grupo submetido ao acesso lateral direto totalizou 127 operados por três cirurgiões distintos.

Não foram incluídos no estudo pacientes com os seguintes critérios:

- diagnóstico de fratura do colo femoral

- pacientes com displasia do quadril tipo Crowe 2,3 e 4
- pacientes submetidos à revisão de artroplastia do quadril

- artroplastia bilateral do quadril

- outro modelo protético não cimentado utilizado

- infecção com subsequente soltura séptica dos componentes

- artroplastia total do quadril tipo híbrida ou tipo cimentada

- acessos minimamente invasivos

- perda de seguimento até nossa análise horizontal ocorrida em junho de 2009

Todos os pacientes foram submetidos à artroplastia total do quadril com componente acetabular MBA ( $L e-$ pine ${ }^{\circledR}$, França) composto por liga de titânio e cobertura de hidroxiapatita e componente femoral tipo Targos (Lepine ${ }^{\circledR}$, França) com geometria taperloc e cobertura de hidroxiapatita. Todas as cabeças modulares tinham diâmetro de $28 \mathrm{~mm}$ confeccionadas de aço. O menor offset femoral é $44,05 \mathrm{~mm}$ e o maior, de $52,09 \mathrm{~mm}$, com variação progressiva de acordo com o tamanho do componente femoral.

$\mathrm{O}$ acesso posterior foi realizado em decúbito lateral conforme técnica descrita por Moore ${ }^{(7)}$, realizando-se a reinserção dos tendões dos músculos rotadores externos (gêmeos, piriforme e obturador interno) com sutura tendínea no tendão do músculo glúteo médio 'em $\mathrm{x}$ ' utilizando-se fio Vycril- $0 \circledR$ e o acesso lateral direto realizado conforme técnica descrita por Hardinge ${ }^{(8)}$, porém com o paciente posicionado em decúbito lateral.

A profilaxia anti-infeciosa foi realizada com cefuroxime $1,5 \mathrm{~g}$ endovenoso a cada 12 horas até 48 horas, assim como foi realizada profilaxia anti-trombótica mecânica e medicamentosa com heparina de baixo peso molecular até 30 dias do período pós-operatório. A fisioterapia ativa assistida foi iniciada no primeiro dia de pós-operatório, evitando-se flexão acima de 90 graus, adução menor que 10 graus em relação ao eixo central do corpo ou qualquer movimento rotacional do membro operado. O treino de marcha, assim como, o de transposição poltrona-leito foi administrado antes da alta, que ocorreu no quinto dia de pós-operatório.

Ambos os grupos foram comparados quanto ao gênero, idade, lado operado e diagnóstico inicial. Eles tiveram seus componentes acetabulares colocados com técnica de estabilidade tipo press-fit like e receberam fixação suplementar com parafusos conforme a preferência do cirurgião. O componente de polietileno utilizado possui um rebordo de 10 graus progressivo, envolvendo $90 \%$ do comprimento da circunferência do componente. 
Foi realizada uma busca por episódio de luxação até o sexto mês de pós-operatório, ou qualquer episódio ocorrido até a análise transversal em junho de 2009, nos pacientes com seguimento maior que seis meses. Além dos dados demográficos e episódios de luxação, foram aferidos a inclinação acetabular e o tempo de seguimento. Consideramos malposicionados os acetábulos com ângulo de abdução menor que 30 graus ou maior que 55 graus e acetábulos com descobertura na zona 1 de DeLee e Charnley maior que $20 \%$.

O tamanho dos componentes acetabular e femoral foi avaliado e dividido em dois grupos, sendo o grupo de offset femoral menor entre $44,05 \mathrm{~mm}$ e $47,11 \mathrm{~mm}$ e o de offset femoral maior entre $50,18 \mathrm{~mm}$ e $52,09 \mathrm{~mm}$. O grupo de acetábulo menor variou entre $46 \mathrm{~mm}$ de diâmetro e $52 \mathrm{~mm}$ e o grupo de acetábulo maior, entre $54 \mathrm{~mm}$ de diâmetro e $60 \mathrm{~mm}$. O tamanho do colo da cabeça de $28 \mathrm{~mm}$ também foi aferido, variando entre menos 3,5mm até mais $7 \mathrm{~mm}$. A análise dos resultados foi feita com revisão dos prontuários do nosso serviço.

Os dados com distribuição normal foram avaliados com testes paramétricos. A comparação entre os grupos foi feita pelo teste $t$, bicaudal, utilizando nível significância $p<0,05$. Os dados qualitativos não numéricos foram tabulados em tabelas de contingência de dupla entrada e foram analisados com o teste do qui-quadrado ou teste exato de Fisher quando necessário. Também foi utilizado nível de significância $\mathrm{p}<0,05$.

\section{RESULTADOS}

Os dados demográficos dos dois grupos de pacientes estão presentes na tabela 1 .

Tabela 1 - Análise estatística dos dois grupos de pacientes: acesso posterior e acesso lateral direto

\begin{tabular}{l|c|c|c}
\hline & $\begin{array}{c}\text { Grupo acesso } \\
\text { posterior }\end{array}$ & $\begin{array}{c}\text { Grupo acesso } \\
\text { lateral direto }\end{array}$ & Valor de p \\
\hline $\begin{array}{l}\text { Gênero } \\
\text { masculino/feminino }\end{array}$ & $58 / 47$ & $56 / 71$ & 0,11 \\
\hline $\begin{array}{l}\text { Lado } \\
\text { direito/esquerdo }\end{array}$ & $54 / 51$ & $60 / 67$ & 0,61 \\
\hline Idade (média) & 53,8 & 52,3 & 0,87 \\
\hline $\begin{array}{l}\text { Diagnóstico } \\
\text { Osteoartrose primária/secundária }\end{array}$ & $31 / 74$ & $48 / 79$ & 0,23 \\
\hline
\end{tabular}

O tempo de seguimento dos dois grupos variou entre seis e 42 meses, com média de 23,7 meses. Observamos um caso isolado de luxação anterior da prótese em um paciente do grupo submetido ao acesso lateral direto $(0,8 \%)$ contra $0 \%$ no grupo de acesso posterior, sem diferença significativa $(\mathrm{p}=1)$.

A luxação ocorreu em uma paciente com diagnóstico de artrite reumatoide de 47 anos de idade, submetida à artroplastia do quadril por acesso lateral direto após três semanas de cirurgia, sendo tratada com redução incruenta sem recidiva da luxação após três anos e um mês de seguimento.

A inclinação do componente acetabular não apresentou diferença significativa entre os grupos, com $96,2 \%$ de bom posicionamento no grupo do acesso posterior e $92,1 \%$ no do acesso lateral direto $(\mathrm{p}=0,27)$.

Não incluímos dois pacientes do grupo do acesso lateral direto e um paciente do grupo do acesso posterior por diagnóstico de infecção profunda com necessidade de revisão dos componentes protéticos.

A tabela 2 apresenta o resumo do tamanho dos componentes da prótese.

Tabela 2 - Componentes da prótese

\begin{tabular}{l|c|c|c}
\hline & $\begin{array}{c}\text { Grupo acesso } \\
\text { posterior }\end{array}$ & $\begin{array}{c}\text { Grupo acesso } \\
\text { lateral direto }\end{array}$ & Valor de p \\
\hline $\begin{array}{l}\text { Componente acetabular } \\
\text { menor/maior }\end{array}$ & $45 / 60$ & $47 / 80$ & 0,44 \\
\hline $\begin{array}{l}\text { Componente femoral } \\
\text { menor/maior }\end{array}$ & $72 / 33$ & $90 / 37$ & 0,81 \\
\hline $\begin{array}{l}\text { Colo } 28 \mathrm{~mm} \\
-3,5 \text { e } 0 \mathrm{~mm} / \\
+3,5 \mathrm{e} 7 \mathrm{~mm}\end{array}$ & $95 / 10$ & $85 / 42$ & $<0,001^{*}$ \\
\hline
\end{tabular}

\section{DISCUSSÃO}

O estudo das duas amostras dos pacientes não evidenciou qualquer viés de seleção. Como se trata de um estudo retrospectivo, não houve randomização, sendo a divisão dos dois grupos decorrente da escolha pessoal do cirurgião quanto ao acesso que está mais habituado a realizar. Porém, devemos salientar a baixa média de idade dos pacientes de ambos os grupos.

Tal achado coincide com a alta proporção de pacientes com osteoartrose secundária, normalmente mais jovens, totalizando $66 \%$ de toda a casuística. Acreditamos que isso se deve à procura de casos mais complexos por nosso serviço, decorrente da própria estruturação do Sistema Único de Saúde pública ${ }^{(9,10)}$.

Obtivemos um caso de luxação no grupo de pacientes submetido ao acesso lateral direto e nenhum caso no grupo do acesso posterior, embora sem significado esta- 
tístico. A prevalência na casuística total (232 pacientes) é de $0,4 \%$, valor este menor que o da maioria dos autores revistos $^{(11-13)}$. Os principais fatores que acreditamos serem os responsáveis por tal fato são: menor média de idade de toda a casuística, exclusão de pacientes com fraturas do colo femoral, utilização de uma prótese com duas características de proteção antiluxação e treinamento e protocolo adequados de reabilitação pós-operatória.

Em uma série anterior, utilizando-se o acesso anterolateral com prótese tipo Charnley realizada pelo mesmo grupo (cabeça de diâmetro de 22mm), observamos 3,4\% de luxação em 115 artroplastias, valores similares também aos de outros autores nacionais ${ }^{(14,15)}$.

A não inclusão de pacientes com fratura do colo femoral na casuística permite maior acurácia estatística em nossa opinião, pois tais pacientes conhecidamente apresentam maior prevalência de luxação por três aspectos, a saber: arco de movimento articular maior que o de pacientes com osteoartrose, maior idade e menor potência muscular. Tais características geralmente elevam a prevalência de valores que podem chegar a cerca de $10 \%{ }^{(9,15)}$.

A prótese utilizada em todos os pacientes apresenta offset maior que o habitual; o menor componente femoral tem offset de $44,05 \mathrm{~mm}$, valor este compatível com os maiores offsets de outros fabricantes. Isso caracteriza, sem dúvida, um fator de maior estabilidade, porém, deve-se atentar quanto ao possível alongamento do membro acima do desejável. Tal fato também explica por que os dois tamanhos de colo mais curtos foram os mais utilizados em ambos os grupos, embora mais frequentemente no acesso posterior.

Tal variação talvez se deva à variação de cirurgiões nos pacientes do grupo do acesso lateral direto, o que caracteriza um viés do nosso trabalho, porém, o mesmo modelo de prótese em todos os pacientes é um fator de enriquecimento científico, não observado na maioria dos trabalhos.

O posicionamento acetabular não apresentou diferença entre os grupos, porém, não realizamos a medida de anteversão acetabular. Acredita-se que o posicionamento é fator importante na gênese das luxações, principalmente no acesso posterior, onde o cirurgião, no início da curva de aprendizado, pode tender à retroversão acetabular ${ }^{(16)}$.

$\mathrm{O}$ treinamento e educação preventivos no período pré e pós-operatórios são de fundamental importância na prevenção das luxações e consideramos que esse foi um dos fatores da nossa baixa prevalência ${ }^{(11,17)}$.

Acreditamos que a maior incidência de luxação no acesso posterior que ocorria no passado devia-se a erro de posicionamento acetabular com tendência à retroversão, assim como a não reinserção dos tendões rotadores externos. Tal fato é confirmado por inúmeros trabalhos recentes apresentados em duas metanálises ${ }^{(3,6)}$.

\section{CONCLUSÃO}

Observamos baixa prevalência dessa complicação nos dois acessos estudados, decorrente de técnica adequada e medidas educativas no período pós-operatório.

\section{REFERÊNCIAS}

1. Charnley J. Arthroplasty of the hip. A new operation. Lancet. 1961;1(7187):1129-32.

2. Sanchez-Sotelo J, Haidukewych GJ, Boberg CJ. Hospital cost of dislocation after primary total hip arthroplasty. J Bone Joint Surg Am. 2006;88(2):290-4.

3. Kwon MS, Kuskowski M, Mulhall KJ, Macaulay W, Brown TE, Saleh KJ. Does surgical approach affect total hip arthroplasty dislocation rates? Clin Orthop Relat Res. 2006;(447):34-8.

4. Berry DJ, von Knoch M, Schleck CD, Harmsen WS. Effect of femoral head diameter and operative approach on risk of dislocation after primary total hip arthroplasty. $\mathrm{J}$ Bone Joint Surg Am. 2005;87(11):2456-63.

5. Ritter MA, Harty LD, Keating ME, Faris PM, Meding JB. A clinical comparison of the anterolateral and posterolateral approaches to the hip. Clin Orthop Relat Res. 2001;(385):95-9.

6. Jolles BM, Bogoch ER. Posterior versus lateral surgical approach for total hip arthroplasty in adults with osteoarthritis. Cochrane Database Syst Rev. 2006;3:CD003828.

7. Moore AT. Metal hip joint: a new self-locking vitallium prosthesis. South Med J. 1952;45(11):1015-9

8. Hardinge K. The direct lateral approach to the hip. J Bone Joint Surg Br. 1982;64(1):17-9.

9. Macedo CAS, Galia CR, Rosito R, Perea CEF, Müller LM, Verzoni GV, et al. Abordagem cirúrgica na artroplastia total primária de quadril: ântero-lateral ou posterior? Rev Bras Ortop. 2002;37(9):387-91.

10. Meek RM, Allan DB, McPhillips G, Kerr L, Howie CR. Epidemiology of dislocation after total hip arthroplasty. Clin Orthop Relat Res. 2006;(447):9-18.
11. Lübbeke A, Suvà D, Perneger T, Hoffmeyer P. Influence of preoperative patient education on the risk of dislocation after primary total hip arthroplasty. Arthritis Rheum. 2009;61(4):552-8.

12. Blom AW, Rogers M, Taylor AH, Pattison G, Whitehouse S, Bannister GC. Dislocation following total hip replacement: the Avon Orthopaedic Centre experience. Ann R Coll Surg Engl. 2008;90(8):658-62.

13. Phillips CB, Barrett JA, Losina E, Mahomed NN, Lingard EA, Guadagnoli E, et al. Incidence rates of dislocation, pulmonary embolism, and deep infection during the first six months after elective total hip replacement. J Bone Joint Surg Am. 2003;85(1):20-6.

14. Vicente JRN, Miranda FG, Bernabé AC, Angelini FJ, Cabrita H, Teochi LF, et al. Artroplastia total do quadril cimentada de Charnley: estudo retrospectivo de 115 casos. Rev Bras Ortop 1998; 33(10):773-6.

15. Aristide RSA, Honda E, Polesello G, Miashiro EH, Reginaldo SS. Luxação da prótese total do quadril em pacientes com fratura do colo do fêmur. Rev Bras Ortop. 1997;32:787-91.

16. Biedermann R, Tonin A, Krismer M, Rachbauer F, Eibl G, Stöckl B. Reducing the risk of dislocation after total hip arthroplasty: the effect of orientation of the acetabular component. J Bone Joint Surg Br. 2005;87(6):762-9.

17. Peak EL, Parvizi J, Ciminiello M, Purtill JJ, Sharkey PF, Hozack WJ, Rothman RH. The role of patient restrictions in reducing the prevalence of early dislocation following total hip arthroplasty. A randomized, prospective study. J Bone Joint Surg Am. 2005;87(2):247-53. 